\title{
Representaciones sociales y comunicación: apuntes teóricos para un diálogo interdisciplinar inconcluso
}

\author{
Social representations and communication: theoretical notes for an \\ interdisciplinary dialogue in progress
}

\author{
Rainer Rubira-García / rainer.rubira@urjc.es \\ http://orcid.org/0000-0002-5667-6080 \\ Universidad Rey Juan Carlos, España \\ Belén Puebla-Martínez / belen.puebla@urjc.es \\ http://orcid.org/0000-0002-1481-4238 \\ Universidad Rey Juan Carlos, España
}

\begin{abstract}
The text makes a critical approach to the concept of social representations and its epistemological value for the understanding of interaction processes, in particular the communication ones. From a bibliographical review of authors, schools, theoretical and methodological perspectives, an attempt is made to systematize the fundamental contributions of the concept of representations and its relation to communication as a field of study. From an interdisciplinary perspective, the points of contact between representations and mediations within communicative dynamics are evaluated and some essential elements are suggested to consider the theoretical importance of social representations in communication research.
\end{abstract}

Key words: representations, communication, mediations, review.

Resumen: El texto realiza una aproximación crítica al concepto de representaciones sociales y su valor epistemológico para la comprensión de los procesos de interacción, en específico los de comunicación. A partir de una revisión bibliográfica documental sobre autores, escuelas, perspectivas teóricas y metodológicas se intenta sistematizar los aportes fundamentales del concepto de representaciones y su relación con el campo de estudios de la Comunicación. Desde una mirada interdisciplinar, se evalúan los puntos de contacto entre las representaciones y las mediaciones dentro de las dinámicas comunicativas y se sugieren algunos elementos esenciales a considerar para valorar la importancia teórica de las representaciones sociales en la investigación en Comunicación.

Palabras clave representaciones, comunicación, mediaciones, crítica. 


\section{Introducción}

El concepto de representaciones sociales remite directamente al punto de unión entre las realidades discursivas (comunicativas) y fácticas, entre la imaginación y la acción, actuando como nexo entre el universo interior y el exterior de los sujetos. Sirven, por tanto, de mapa para la interpretación del mundo, para interactuar con él. Interactuar, por ejemplo, a través de procesos de comunicación, entre otros. De tal modo, las interacciones entre los sujetos, los objetos de conocimiento y el contexto de interpretación y acción se erigen como una de las preocupaciones epistémicas centrales para la teoría de las representaciones sociales bajo una mirada holística, que, sin duda, es uno de sus aportes esenciales dentro de las ciencias sociales en el siglo XX.

Las representaciones son un producto y, al mismo tiempo, son el proceso por el cual dicho producto tiene lugar. De tal suerte, una representación puede ser estudiada como producto y como proceso.

Los investigadores, no obstante, se han centrado en los contenidos de la representación, en el producto, en tanto la deconstrucción del proceso representacional se ha revelado difícil en extremo. A pesar de lo anterior, muchos autores, entre ellos Denise Jodelet (1986) y Tomás Ibáñez (1988), abogan por no olvidar la naturaleza procesal y por tanto interactiva y comunicativa de toda representación, incluso cuando se analiza su estructura formal y los elementos que la integran.

La representación social es, a la vez, pensamiento constituido y pensamiento constituyente. En tanto que pensamiento constituido, las representaciones sociales se transforman efectivamente en productos que intervienen en la vida social como estructuras preformadas a partir de las cuales se interpreta, por ejemplo, la realidad. Estos productos reflejan en su contenido sus propias condiciones de producción, y es así como nos informan sobre los rasgos de la sociedad en la que se han formado. En tanto que pensamiento constituyente, las representaciones sociales no sólo reflejan la realidad sino que intervienen en su elaboración. [...] La representación social constituye en parte el objeto que representa. No es el reflejo interior, es decir, situado en la cabeza de los sujetos, de una realidad exterior, sino que es un factor constitutivo de la propia realidad (Ibáñez, 1988: 36).

A partir de una sistematización bibliográfica-documental crítica, en este trabajo abordaremos las distintas perspectivas de estudio del concepto de representaciones sociales, las conexiones de éste con los procesos de interacción social -en específico de comunicación, sobre todo teniendo en cuenta los aportes iberoamericanos en ese sentido-, los distintos tipos de representaciones, sus funciones e importancia para las dinámicas comunicativas, así como las críticas fundamentales que ha recibido esta propuesta teórica. 
El análisis sistemático bibliográfico-documental implica la producción de "un documento escrito que recoja un caso lógicamente argumentado y basado en una comprensión global del estado actualizado de conocimiento sobre un tema de estudio" (Machi y McEvoy, 2016: 5).

La investigación sistemática bibliográfica-documental que proponemos pasa por una serie de etapas consensuadas, a saber: búsqueda de la literatura, almacenamiento, síntesis, análisis y presentación del mismo (Booth et al., 2016: 2). En esta investigación se han trabajado autores, escuelas, perspectivas teóricas y metodológicas dentro del campo de estudio de las representaciones sociales sin una pretensión omnicomprensiva o totalizante, sino, por el contrario, más bien reflexiva e interpretativa.

\section{Perspectivas teóricas y metodológicas en el estudio de las representaciones sociales}

La noción de representaciones puede inscribirse en el marco general de la Sociología del Conocimiento tal y como la entienden Berger y Luckmann (1986). Las representaciones construyen el objeto al cual se refieren y, en ese sentido, modelan la realidad social. En ningún momento, sin embargo, se debe olvidar que están mediadas por la misma realidad que las circunda.

La realidad siempre actúa a través de su interpretación por los seres sociales. No hay más realidad que la realidad tal y como la desciframos. Son los significados que le atribuimos los que van a constituirla como la única realidad que para nosotros existe efectivamente. La realidad tal y como la interpretamos es la única realidad que puede tener, por consiguiente, unos efectos sobre nosotros (Ibáñez, 1988: 26).

Es posible identificar al menos cuatro vertientes que han desarrollado posiciones teóricas particulares y consecuentemente metodológicas en el campo de las representaciones: la escuela clásica, la estructural, la perspectiva sociodinámica de Ginebra y el enfoque de Wagner y Hayes (2005). Además son importantes los aportes de Robert Farr (Farr y Moscovici,1984), en el Reino Unido, y de María Auxiliadora Banchs (1984 y 1990) desde Venezuela. Sin pretender ser omnicomprensivos en nuestro trabajo, la discusión sobre estas escuelas cubre diferentes perspectivas: desde lo cualitativo hasta lo cuantitativo, desde el objeto hasta el proceso.

La escuela clásica, de inspiración psicosocial, tiene su asiento en París y constituye el núcleo duro de la teoría de las representaciones sociales, iniciada por Serge Moscovici en 1961 y perfilada en detalle por su discípula Denise Jodelet durante los años subsiguientes. 
De hecho, ha sido Jodelet (1986) quien ha logrado la que tal vez sea la definición más aceptada de representación social hasta el momento y también quien ha concretado y perfeccionado muchas de las ideas esenciales de Moscovici. Las investigaciones de este último y sobre todo de Jodelet se han basado en el enfoque cualitativo a partir del análisis de los discursos y las prácticas sobre el objeto de la representación.

Tanto Jodelet como Moscovici entienden que las representaciones se evidencian en el lenguaje y en las acciones sociales de grupos específicos, y remarcan la importancia del contexto de comunicación. En particular, los medios de comunicación de masas y las instituciones son considerados los espacios de desarrollo por excelencia de las representaciones en la vida cotidiana.

Los dos han privilegiado el uso de cuestionarios abiertos y de entrevistas en profundidad, así como de la observación participante para descubrir el universo simbólico donde los sujetos construyen sus representaciones sobre un objeto específico.

Por su parte, la escuela estructural, de esencia psico-cognitiva, también se localiza en Francia pero no en París, sino en la sureña Aix-en-Provence. Se trata del denominado Grupo de MIDI que dirige Jean-Claude Abric con la colaboración de Claude Flament (2001).

Los estudios de estos investigadores se caracterizan por el interés en la composición de las representaciones sociales con énfasis en los procedimientos de cognición. Tomando como punto de partida a Moscovici, han desarrollado la hoy reconocida Teoría del núcleo central que, sobre la base de los principios del estructuralismo, intenta explicar la manera en que se organiza una representación.

Según Abric (2001), las representaciones son fenómenos sociocognitivos, cuya peculiar naturaleza, constituida por un núcleo central y un sistema periférico, les permite ser estables y flexibles al mismo tiempo. El Grupo ha insistido en los diseños experimentales y cuantificables, con lo cual ha realizado indudables aportes al campo de las representaciones sociales en su conjunto, aunque el empleo de la doctrina metodológica de matiz positivista le ha provocado un buen número de críticas.

La perspectiva sociodinámica de Ginebra está encabezada por Willem Doise (1985), quien ha hecho hincapié en el estudio de las representaciones como espacios de intercambio simbólico. Para comprender una representación hay que analizar las relaciones que se establecen entre el metasistema social y el universo mental de los sujetos, los cuales se encuentran afectados por la respectiva posición que ocupan en dicho metasistema. 
La particular pertenencia social de individuos y grupos es determinante en la estructuración de sus representaciones. Así, las investigaciones de Doise (1991 y 1993) se distinguen por el uso de métodos estadísticos correlacionales. Con una fuerte inspiración sociológica, la perspectiva de Ginebra ha centrado su interés en las condiciones de producción y circulación de las representaciones.

Para Doise (1985) no es posible definir a las representaciones en términos de consenso, pues en última instancia éstas son tomas de posición individuales. En las representaciones lo consensuado sería, en todo caso, el mecanismo o principio organizador que articula a la representación social.

El enfoque de Wagner, desde la Universidad de Linz, se concentra en resaltar el carácter construido de las representaciones. "En lugar de imaginar a las representaciones dentro de las mentes es mejor imaginarlas a través de las mentes, como si fueran una especie de red tejida por las personas mediante sus conversaciones y sus acciones" (Wagner et al., 1999: 96).

La propuesta de Wagner, desde una postura culturalista, entiende que las representaciones sociales, aunque se manifiestan en expresiones individuales, evidencian el consenso que las caracteriza no en un sentido estadístico, sino en el ámbito de lo epirracional.

$\mathrm{Al}$ respecto, explica que no es necesario que dos personas compartan determinadas creencias (o representaciones) sobre un tema para que se comuniquen; sólo deben compartir un sistema epirracional de proposiciones posibles o admisibles independientemente de si están de acuerdo o no con ellas.

Es preciso apuntar también como un referente significativo de la teoría de las representaciones sociales a Robert Farr, profesor británico de la London School of Economics durante dos décadas. Farr centró su trabajo en los vínculos entre representaciones, sentido común y conocimiento científico fundamentalmente. Según Farr (Farr y Moscovici, 1984: 496), las representaciones son:

Sistemas cognoscitivos con una lógica y un lenguaje propios. No representan simplemente "opiniones acerca de", "imágenes de", o "actitudes hacia" sino "teorías o ramas del conocimiento" con derechos propios para el descubrimiento y la organización de la realidad. Sistemas de valores, ideas y prácticas con una función doble: primero, establecer un orden que permita a los individuos orientarse en su mundo material y social y dominarlo; segundo, posibilitar la comunicación entre los miembros de una comunidad proporcionándoles un código para el intercambio social y un código para nombrar y clasificar sin ambigüedades los diversos aspectos de su mundo y de su historia individual y grupal. 
Por último, hay que mencionar a María Auxiliadora Banchs (1984 y 1990), quien no sólo ha sido una de las profesoras con mayor peso en la divulgación de la perspectiva de estudio de las representaciones sociales en español, sino que también ha sido una de las defensoras más acérrimas de la interpretación social e histórica y, por tanto, procesual del concepto.

\section{Representaciones, interacción social y comunicación}

La idea de representaciones constituye un elemento teórico indispensable para la reflexión sobre los procesos de comunicación social y está directamente vinculada al término de mediaciones de la perspectiva latinoamericana de investigación en el campo de estudios de la Comunicación. Cada vez más se tiene en cuenta en estudios de apropiación de la ciencia y la tecnología, como pueden ser los de representaciones sociales sobre nanotecnología (Bertoldo et al., 2016; Brunel et al., 2017) o los de representaciones sociales sobre robótica (Piçarra, 2016). Incluso encontramos investigaciones sobre representaciones sociales del ciberacoso en adolescentes (Young et al., 2016) y de representaciones sociales sobre la enseñanza en línea (Islas, 2016).

Las representaciones sociales sirven de vehículo de enlace, de tejido conectivo entre el saber y el hacer, entre cognición y acción, y entre sujeto y objeto. Surgen en medio de esas interacciones y se erigen en una mediación significativa a considerar en torno a las mismas. Más importante aún, Moscovici (1985) subraya el hecho de que las representaciones tienen carácter recursivo, es decir, se retroalimentan continuamente de las acciones y conocimientos individuales, en una dialéctica de la cotidianidad que en cierta medida implica un avance respecto a la argumentación positivista en ciencias sociales.

El sujeto es, por tanto, un agente activo en la configuración de las representaciones sociales, como lo son también el contexto y los propios objetos de conocimiento. En este punto, la perspectiva de las representaciones conecta directamente con la revolución que trajo el paradigma culturalista al campo de la comunicación en América Latina con Jesús Martín-Barbero (1987) y su antológico libro De los medios a las mediaciones.

Las representaciones facilitan la producción y reproducción de realidades sociales en buena medida, porque ellas mismas son el resultado de esas realidades. "Así, por ejemplo, las comunicaciones sociales serían difícilmente posibles si no se desenvolvieran en el contexto de una serie, suficientemente amplia, de representaciones compartidas" (Ibáñez, 1988: 43). 
Desde el interaccionismo simbólico, Mead (en Da Silva, 2011) y Blummer (1969) suponen que la configuración de representaciones sociales tiene su origen en interacciones cotidianas, como es el caso de los intercambios comunicativos. Moscovici añade a esa idea que las representaciones pueden emerger, mediante procesos de observación o de reflexión individuales, aunque las redes de comunicación siguen siendo las fuentes más importantes de representaciones entre grupos o comunidades de individuos.

Moscovici precisa que las representaciones sociales responden a tres rasgos principales:

a) Criterio cuantitativo: señala el grado de extensión que pueden alcanzar en una comunidad.

b) Criterio productivo: indica su capacidad de emerger y actuar desde una organización social.

c) Criterio funcional: destaca el rol que juegan en la formación y orientación de las conductas y las comunicaciones (en Banchs, 1984: 5).

En tanto teorías del sentido común, algo en lo que coinciden Moscovici, Jodelet, Abric y Banchs, entre otros autores, las representaciones sociales son útiles para establecer las visiones de un grupo sobre determinados objetos de conocimiento con los que interactúa. "Theoría $(\theta \varepsilon \omega p i \alpha)$ es ver y entender, contemplar precisamente aquello que está ocurriendo en el origen” (Zubiri, 1994: 41). Las representaciones intervienen en las relaciones entre individuos y sus objetos formando parte de una triada de sentido que se retroalimenta de forma dialéctica.

Las representaciones son teorías o representan el papel de tales. Por consiguiente, en esta cualidad deben mostrar "cómo suceden las cosas". Dicho de otra manera, las representaciones tienen por misión: primero, describir; después, clasificar, y por último, explicar (he aquí por qué las representaciones incluyen las denominadas "teorías implícitas" que sirven únicamente para clasificar a personas o comportamientos, y los esquemas de atribución destinados a explicarlas) (Moscovici y Hewstone, 1986: 699).

Las representaciones sociales aparecen además conectadas con otras representaciones en sistemas complejos que responden a las historias individuales y colectivas de los sujetos y también a las interacciones de éstos con los objetos de conocimiento.

Las representaciones están inscritas en los pliegues del cuerpo, en las disposiciones que tenemos y en los gestos que realizamos. Forman la sustancia de ese habitus del que hablaban los antiguos, que transforma una masa de instintos y órganos en un universo ordenado, en un microcosmos humano del macrocosmos físico, hasta el punto de hacer que nuestra biología aparezca como una sociología y una psicología, nuestra naturaleza como una obra de la cultura. Enraizada así en el cuerpo, la vida de las representaciones se revela como una vida de memoria (Moscovici y Hewstone, 1986: 708-709). 
Manuel Martín-Serrano (2004), investigador español en Comunicación de la llamada Escuela de Madrid, ha trabajado con bastante frecuencia las representaciones como componente del sistema de comunicación. Junto con los actores, las expresiones y los instrumentos, éstas son parte del modelo dialéctico para el estudio de los sistemas de comunicación que él formula en su obra Teoría de la Comunicación. Epistemología y Análisis de la Referencia. Para este autor:

Una representación social consiste en la propuesta de una determinada interpretación de lo que existe o de lo que acontece en el entorno. La representación social hace referencia precisamente a tales o cuales temas, incluyendo unos datos en vez de otros y sugiriendo ciertas evaluaciones en vez de otras posibles. Cuando el relato es elaborado por un mediador institucional (institución mediadora) y está destinado a una comunidad, la representación social puede llegar a adquirir el valor de una representación colectiva o se legitima por ella. [...] La representación social es una interpretación de la realidad que está destinada a ser interiorizada como representación personal por determinados componentes de un grupo. En consecuencia, la representación social tiene que estar propuesta en un relato susceptible de ser difundido. [...] La representación social deviene un producto cognitivo inseparable del producto comunicativo, entendiendo por "producto comunicativo" un objeto fabricado que tiene un valor de uso concreto: poner la información que han elaborado unos sujetos sociales a disposición de otros (Martín-Serrano, 2004: 57).

Como es lógico, las disquisiciones de Martín-Serrano están muy enfocadas a la comunicación masiva y, en particular, a la prensa.

\section{Clasificación de las representaciones sociales}

Según Moscovici (1985), existen tres tipos de representaciones sociales en lo fundamental: emancipadas, polémicas y hegemónicas.

Las representaciones emancipadas no tienen el alcance macro de las hegemónicas. Son propias de grupos que surgen con nuevas visiones y criterios del mundo, trayendo consigo la semilla de un posible cambio. En esencia, éstas son muy flexibles y dinámicas. Con el tiempo, a veces evolucionan hasta convertirse en representaciones hegemónicas de forma lenta y pacífica.

En la mayoría de los casos, sin embargo, las representaciones emancipadas se transforman en representaciones polémicas, las cuales se configuran en torno a hechos u objetos de relevancia que constituyen el centro de conflictos intergrupales.

En parte, las representaciones polémicas son el resultado del antagonismo manifiesto entre grupos específicos. Como consecuencia del enfren- 
tamiento entre distintas representaciones polémicas, en un momento dado puede producirse un salto histórico.

Las representaciones hegemónicas se distinguen por su elevado nivel de consenso, gracias a lo cual juegan un papel muy destacado en las prácticas sociales de los sujetos.

A causa de sus altos niveles de homogeneidad y estabilidad, esta clase de representación, de prolongada sedimentación, es propia de los fenómenos de identidad y actúa como un mapa que guía, casi de modo inconsciente, el comportamiento de comunidades, etnias y naciones enteras. Su naturaleza un tanto determinista la acerca a la representación colectiva de Durkheim.

Estos tres tipos de representaciones se relacionan directamente con los procesos de lectura e interpretación definidos por Stuart Hall (1993). En la lectura negociada, el receptor pacta con el emisor valores y significados del mensaje, en una dinámica de convergencia. La lectura oposicional implica un rechazo frontal y consciente del receptor en relación con el mensaje, mientras que la lectura preferente responde a una aceptación hegemónica del mismo.

Una representación se confirma siempre en relación con otras representaciones, en especial, con aquellas que sobre el grupo y su propia postura tienen los demás. Cada representación, en tanto sistema complejo, no permanece aislada del resto de las representaciones a su alrededor, así como los grupos de personas no permanecen químicamente puros en la realidad. A veces, las representaciones no se articulan en torno a un objeto unívoco. En ese sentido, Flament (en Abric, 2001) ha definido dos grandes tipos de representaciones: las autónomas y las no autónomas.

Las representaciones autónomas se organizan en torno a un objeto nada más, aunque pueden guardar vínculos estrechos con otras representaciones próximas.

Las representaciones no autónomas, en cambio, son más globales e integran un objeto con otros distintos, concatenados o no, a pesar de que las imágenes aparecen dispuestas dentro de un mismo núcleo central, siguiendo la denominación de la Escuela de Aix-en-Provence.

\section{Representaciones sociales y mediaciones en el proceso comunicativo}

Una representación se distingue por ser prescriptiva y descriptiva, e intenta superar la dicotomía entre individuo y sociedad (Castorina y Barreiro, 2012). Aunque sólo se manifiestan de forma simultánea en las representaciones sociales, vale aclarar que ambos rasgos no son privativos de éstas según Flament (2001), ni el prescriptivo como ha demostrado la Teoría de 
los Scripts (Tomkins, 1962), ni el descriptivo, de acuerdo con la Teoría de la prototipicidad (Rosch, 1977).

El aspecto prescriptivo de la representación es muy complejo y se da en el propio vínculo que establece con las conductas y prácticas relacionadas. La prescripción dice lo que se debe, lo que se puede hacer y lo que no, similar a uno de los imperativos categóricos de Kant. Como contraparte, la propiedad descriptiva de las representaciones es mucho más habitual y evidente que la prescriptiva. La mayoría de las veces, sin embargo, se combina con esta última.

En ocasiones, al describir un objeto, en esencia, estamos dando nuestra percepción de cómo debe ser utilizado y para qué sirve, pues es difícil separar el fin utilitario de los objetos de sus características físicas básicas. Por ejemplo, cuando pensamos en un avión como un objeto autopropulsado, con alas y motores, que recorre grandes distancias, está claro que en el fondo tenemos en mente que se emplea para el transporte entre sitios bastante alejados.

Todas las representaciones cumplen una importante función dentro de los procesos de comunicación, en especial en el mundo de hoy, donde se hace tan difícil digerir las diferentes dimensiones de una realidad en extremo cambiante y parcelada. "La comunicación no es posible sin la participación de las representaciones” (Martín-Serrano, 2004: 32).

Los intercambios simbólicos de la cotidianidad, más que códigos y subcódigos comunes, requieren un basamento de criterios, opiniones, esquemas, valoraciones y reflexiones que sustente y alimente el flujo comunicativo. Cada representación nace al calor de la comunicación, entre las conversaciones de los sujetos y la agenda temática de los media, para al fin y al cabo convertirse en el horno y el combustible que impulsa a la comunicación misma.

Según el investigador mexicano Raúl Fuentes-Navarro (2001: 241), “en las representaciones de los sujetos se puede observar de manera más inmediata la apropiación construida del recurso y los esquemas operativos de la actividad (sea ésta el entretenimiento, el trabajo, la socialización, el aprendizaje, etcétera)". Actividades que en buena medida tienen un componente comunicativo esencial en la contemporaneidad.

Las representaciones, además, integran las novedades que aparecen en el panorama social, con lo cual contribuyen al equilibrio entre los universos subjetivo y objetivo de los seres humanos. De tal suerte, una representación, en tanto estructura dinámica y a la vez estable, facilita la transformación progresiva de las mentalidades.

Los sujetos se desenvuelven en grupos. Sin un repertorio consensuado de ideas, imágenes, consideraciones, la cohesión colectiva en una sociedad sería casi imposible. 
En ese sentido, las representaciones intervienen en la conformación de las identidades sociales e individuales, a veces limando asperezas; otras originando enfrentamientos, sí, pues recordemos que la unidad de un grupo se da siempre en oposición, más o menos violenta, a los demás.

De ese modo, las representaciones configuran el entorno del individuo y en buena medida lo encadenan al estamento que ocupa en la sociedad. Por eso, algunos autores (Ibáñez, 1988: 55) argumentan que, igual que las ideologías, las representaciones permiten legitimar el status quo imperante.

Las representaciones son también una especie de motor que genera la asunción de posturas. Como guía para la actuación de los sujetos, las personas reaccionan en un determinado espectro de posibilidades que enmarca la representación.

Pero el modelo de la reacción de la persona no es del tipo estímulo-respuesta, sino que, como hemos visto, el estímulo está construido en parte por las exigencias de la propia respuesta. El significado que se atribuye al estímulo está orientado por el tipo de respuesta que se está dispuesto a dar. Las representaciones sociales producen los significados que la gente necesita para comprender, actuar y orientarse en su medio (Ibáñez, 1988: 55).

Esto último es aplicable, asimismo, a la comunicación, como práctica que es también en definitiva. Para Denise Jodelet (1986: 486), las representaciones tienen tres funciones básicas: 1) cognitiva de integración de la novedad, 2) de interpretación de la realidad y 3 ) de orientación de las conductas y las relaciones sociales.

Por su parte, Abric (2001) enumera cuatro funciones esenciales de cualquier representación:

a) Función de saber: las representaciones sirven para entender y explicar la realidad. Facilitan la asimilación de los objetos que tocan a la puerta de los grupos al presentarlos de forma comprensible y accesible. Permiten el intercambio social, la transmisión y difusión del conocimiento de sentido común, por lo que se erigen en condición necesaria de la comunicación.

b) Función de identidad: las representaciones son clave para entender los fenómenos de identidad y especificidad de los grupos. Sitúan a los sujetos en el espacio social al constituir parte de la malla invisible que liga a unos individuos con otros. Así, son una garantía de la coherencia y permanencia de la sociedad en el tiempo.

c) Función de orientación: las representaciones conducen los comportamientos sociales como mapas de orientación de las prácticas diarias de las personas. Intervienen en la dilucidación de la finalidad de las situaciones que protagonizan los grupos y en la creación de expectativas y anticipaciones hacia el objeto. 
d) Función justificadora: las representaciones sancionan y legitiman las acciones de los sujetos como válidas, al estar en plena sintonía con el espectro de normas y valores defendidos por los miembros de un grupo.

En ese sentido, las representaciones sociales constituyen entidades de mediación a tener en cuenta. Siguiendo el criterio de Manuel Martín-Serrano (2004: 23), la noción de mediaciones "pretende ofrecer un paradigma adecuado para estudiar todas aquellas prácticas, sean o no comunicativas, en las que la conciencia, las conductas y los bienes entran en procesos de interdependencia”.

Por su parte, para Martín-Barbero (2001:73-74), quien cada cierto tiempo renueva la manera de formular qué son las mediaciones, éstas constituyen, según una de sus últimas conceptualizaciones, "ámbitos que se interponen entre la configuración social y la naturaleza de la comunicación”.

Considerando el conjunto de mediaciones que trabaja Martín-Barbero, como la cotidianidad familiar, la temporalidad social, las competencias culturales, los géneros y el habitus, entre otras, que para él se reproducen en y desde las interacciones comunicativas, es evidente cómo la subjetividad sirve para interpretar y re-construir los fenómenos de comunicación social en la contemporaneidad como puente entre el mundo interior y exterior de las audiencias.

Las representaciones constituyen un sistema mediado y mediador que conecta a los individuos con los objetos sociales en ciertos contextos. Las representaciones en tanto estructuras subjetivas sintetizan diferentes mediaciones del intercambio comunicativo al estructurarse en mapas desde los cuales se le otorga sentido a la cotidianidad.

En la línea de Martín-Barbero, Guillermo Orozco (2002: 26) también ha actualizado en numerosas ocasiones su idea de mediación. En general, este autor mexicano considera que "las mediaciones hay que entenderlas como procesos estructurantes que provienen de diversas fuentes, que inciden en los procesos de comunicación y conforman las interacciones comunicativas de los actores sociales".

Manuel Martín-Serrano (2004: 135-136) argumenta cómo la representación social puede tener tres usos independientes dentro de los procesos comunicativos que, a veces, se solapan. Según este autor, las representaciones pueden ser:

a) Modelos para la acción: pues dan a las informaciones circulantes un sentido que afecta el comportamiento de los individuos.

b) Modelos para la cognición: pues otorgan a esas informaciones significados que permiten construir un conocimiento particular del mundo. 
c) Modelos para la intención: al dotar a las informaciones aprehendidas de un valor normativo y una finalidad prescriptiva.

De acuerdo con el propio Moscovici (1985: 21), su enfoque de representaciones sociales "presupone una mediación constante". Por supuesto, al ubicarse en el punto de contacto entre los imaginarios y las prácticas, como guía socializada para la acción y la comunicación de los sujetos, el constructo de la representación nos ayuda a entender el sistema de mediaciones que afecta cualquier espacio comunicativo.

En su trabajo "Sobre el potencial teórico de las representaciones sociales en el campo de la comunicación”, Rodríguez (2009) nos dice que a pesar de que en los últimos años se evidencia un marcado interés por vincular el concepto de representaciones sociales a los estudios de comunicación, éste no ha sido un asunto prioritario en la agenda investigativa dentro del campo.

\section{Críticas más importantes a la teoría de las representaciones sociales}

Varias han sido las críticas efectuadas en contra de la teoría de las representaciones. Algunos detractores han planteado que el concepto de representación no aporta nada nuevo en comparación con otros como los de actitud e imagen, por ejemplo, sino que contribuye con más confusión y redundancia.

Otra objeción frecuente que también adelantamos con anterioridad se refiere al problema del consenso que se dice promueven las representaciones y el conjunto de consecuencias que esto trae consigo. La idea del consenso puede enmascarar la naturaleza agónica de la sociedad y, en especial, la reproducción de los procesos de dominación y control a través de los cuales se somete a los sujetos para que acepten el status quo legitimado por los grupos de poder.

Hemos explicado que el consenso en representaciones sociales incluye mecanismos de disenso sin los cuales no sedimentaría ni siquiera el núcleo del campo de la representación. Las contradicciones dentro de una representación se justifican en virtud de su estructura compleja, activa y estable a la vez.

Asimismo, se ha comprobado la existencia de representaciones contrapuestas entre grupos enfrentados por el mismo objeto, lo cual indica que el consenso del que hablan Moscovici y sus seguidores no es totalizante.

Así, la preocupación de Bourdieu, Chamboredon y Passeron (Bourdieu et al., 1968), expresada en El oficio del sociólogo, en 1968, acerca del carácter conservador e ingenuo de las filosofías sociales del consenso, incluida la noción de representación, fue rechazada de modo eficaz para el caso que nos ocupa. 
Por su parte, los ataques de Potter y Wetherell, recogidos por Tomás Ibáñez (1988: 73-76), se orientan a dos aspectos centrales de la definición de representaciones: el inevitable anclaje de éstas al grupo dentro del cual se originan y manifiestan, y su condición de mapas-guías de las conductas humanas. ¿Cómo se verifica la existencia de un grupo? Por las representaciones que comparte. ¿Cómo se determina la presencia de una representación? Por la relación unívoca que establece con el grupo.

Esta circularidad tautológica sería, de ser cierta, una deficiencia mortal de la noción de representaciones, pero tiene importantes fisuras a considerar. Primero, los grupos no sólo se verifican por sus representaciones comunes. Hay otros factores como la percepción externa sobre su propia condición de colectividad que no podemos olvidar.

Los grupos son -aunque no en exclusivo- una construcción y no existen solamente por sí mismos; existen, además, en oposición al resto. Incluso, hay grupos que tienen conciencia de tales en tanto son percibidos como grupos por la sociedad. Segundo, la representación no existe nada más en y a través del grupo, sino también por el objeto al cual se refiere. Tercero, los hombres no pertenecen a un único grupo, sino a una red social intrincada $\mathrm{y}$, de hecho, tampoco hay representaciones puras, sino sistemas de representaciones en constante interacción y desarrollo, de lo que se deduce que no es posible aislar una cadena grupo-representación como para encontrar una relación determinante en ese sentido. El punto clave del asunto está en que las representaciones son un proceso y no un producto, aunque se les suela confundir y se piense que son estructuras acabadas e inertes.

En cuanto a las funciones que cumplen las representaciones como mapas para la acción social, Potter y Wetherell (en Ibáñez, 1988) han presentado sus dudas acerca de que una misma representación sea capaz de responder a la infinitud de situaciones potenciales con las cuales se puede topar un sujeto, según varían los contextos y las dinámicas con los demás individuos en el entorno. En el fondo de esta crítica se nota que ambos están pensando de nuevo en la representación como un producto y no como un proceso. Por supuesto, las representaciones no son un generador de respuestas preconcebidas, tan sólo ofrecen un índice, un marco de elección que el propio sujeto ordena.

Desde otra arista, Rom Harré (2002), en un interesante escrito, ha alertado sobre la manera de entender lo social en una representación. Para Harré hay que distinguir las pluralidades distributivas de las colectivas. En una pluralidad distributiva basta con que los sujetos compartan un atributo cualquiera para que éste sea considerado factor común de unidad. 
Si los sujetos se miran como unidades aisladas, encontramos el atributo que es factor común en ellos sin dificultad alguna. En cambio, las pluralidades colectivas se sustentan en el criterio de que los atributos comunes no solamente son compartidos, sino además construidos; por lo tanto, al separar a los sujetos del grupo que los cobija no es posible encontrar en cada uno de ellos por separado los atributos que poseen en el grupo como un todo. Para que una representación sea social debe constituirse mediante una pluralidad colectiva.

Lo anterior a veces se obvia; no por gusto Moscovici ha insistido siempre en que una representación sólo se mantiene por la constante interacción entre el sujeto individual, el sujeto social y el objeto. Las representaciones se producen y reproducen entre los miembros de un grupo, lo cual garantiza su socialidad.

En específico, el construccionismo ha reclamado que las representaciones no son más que una elaboración subjetiva a pesar de lo que se haya dicho al respecto. Siguiendo esta lógica, la representación únicamente se muestra en tanto discurso sobre cualquier objeto de interés para un grupo.

No debemos ignorar que para el construccionismo la realidad es precisamente una construcción humana. De tal suerte, nada existe fuera de la voluntad de los hombres: ni la pobreza ni las enfermedades ni las catástrofes, pues lo relevante es la visión que los individuos reflejan de estos fenómenos.

Durante un tiempo el construccionismo sedujo por lo atrevido de su propuesta, y su crítica en el campo de las representaciones tuvo cierto impacto a pesar de que ésta nunca convenció a Moscovici ni a Jodelet ni a los teóricos principales de la representación. Después de un intenso debate en revistas y otras publicaciones especializadas, las representaciones han demostrado el vacío sobre el que se sostiene la diatriba construccionista.

Aun cuando son más abundantes las investigaciones descriptivas en representaciones por disímiles cuestiones (tiempo, dinero, complejidad del objeto de representación, etc.) esto no implica que una representación social sea un mero producto discursivo. La verbalización es una parte importante, pero no la única dentro de una representación, algunos de cuyos elementos no son ni tan siquiera conscientes.

Sin embargo, no hay por qué ocultar el sustrato subjetivo -en el sentido activo del término- de cualquier representación, sin olvidar el peso de los muchos factores de la realidad que intervienen también en su definición y uso.

El hecho de asumir el carácter subjetivo de las representaciones sociales no niega sino que reafirma su compromiso con la realidad social, sólo que esta realidad social se expresa en las representaciones sociales de forma subjetiva, no como reflejo objetivo de lo representado, que es precisamente a donde va dirigida la crítica del construccionismo social (González-Rey, 2002: 116). 
Ahora, sin dudas, un buen porcentaje de los ataques en contra de las representaciones se han enfocado hacia un área capital, en verdad bastante desatendida: las herramientas metodológicas para su investigación. A la evidente imposibilidad de medir, en términos cuantificables, la configuración de las estructuras representacionales -un requisito que exige el positivismo dominante-, se agrega el peligro de la opción cualitativa a mano: que los discursos verbalizados de los sujetos estén demasiado contaminados como para servir de material de análisis.

La solución hasta ahora consiste en aplicar instrumentos exhaustivos que deben cruzarse luego entre sí con vistas a disminuir la posibilidad de engaños o sesgos involuntarios en los datos. Desmontar los discursos de los sujetos no parece suficiente para llegar a entender una representación; es imprescindible el uso de la etnografía profunda. Así, se ha argumentado que las representaciones son una falacia metodológica, un invento que surge de los propios instrumentos que emplean los investigadores para su estudio. La teoría de las representaciones sociales sería una teoría Pigmalión construida con base en los hallazgos que pretende mostrar como propios.

\section{Conclusiones}

Ha sido difícil para los científicos sociales liberarse de las limitaciones del paradigma positivista, aun cuando nominalmente lo hayan rechazado. Aceptar la complejidad inherente al concepto de representación como tejido constituido y constituyente, mediado y mediador del pensamiento y la práctica cotidianos de un grupo particular no es sencillo, menos si continuamos mirando al mundo con prismas graduados para eventos de carácter más cerrado y puntual. De esa forma siempre nos quedaremos en la superficie sin ir más allá, a la esencia. De hecho, en representaciones sociales el uso de metodologías activas es extremadamente favorable para comprender la producción del conocimiento de los sujetos (De Castro, 2016).

En el que probablemente sea su último libro publicado en vida, Serge Moscovici, en colaboración con Miles Hewstone (Moscovici y Hewstone, 2013), nos dice en Le scandale de la pensée sociale que la teoría de las representaciones sociales es, ante todo, un intento de buscar las intersecciones de la comunicación, la ciencia y, sobre todo, de la cultura con el conocimiento de una sociedad.

Aún con mucho que avanzar en el perfeccionamiento de las herramientas de investigación en representaciones sociales y su importancia para comprender los procesos comunicativos, varios autores han reafirmado la 
pertinencia de la perspectiva cualitativa para su estudio, como bien nos comenta Fernando González-Rey (2002) en Sujeto y subjetividad: una aproximación histórico-cultural.

La trascendencia del modelo de representación social para el campo de las ciencias de la comunicación puede resumirse en los siguientes puntos, sin pretensiones de agotar todos ellos:

a) Insiste en la dimensión social del universo subjetivo y en cómo este no es innato, pues parte de una interiorización de las ideologías, la cultura y las prácticas, en línea con las aportaciones de la perspectiva latinoamericana de estudios de comunicación.

b) Articula los procesos cognitivos con los procesos grupales e intergrupales más generales, lo cual puede resultar muy importante para investigar los procesos de convergencia actuales en el ecosistema comunicativo.

c) Intenta examinar el proceso de conocimiento de sentido común, desarrollando concretamente el programa de investigación planteado por sociólogos, etnometodólogos y fenomenólogos desde una perspectiva interdisciplinar muy apropiada para el análisis de la comunicación desde un enfoque holístico.

d) Al plantear la relación entre sujeto individual, sujeto social y objeto como lectura ternaria en el sentido de Pierce (1986), articula aspectos psicológicos y sociológicos que ofrecen una visión más global y menos parcializada de las interacciones cotidianas, incluidas las comunicativas.

e) Constituye una alternativa metodológica de matiz psicosocial que puede enriquecer las tendencias crítica y culturalista que han dominado el campo de estudios de la comunicación durante las últimas décadas.

Las representaciones sociales como elaboración conceptual ofrecen una gran riqueza epistemológica a la hora del análisis de los procesos de comunicación contemporáneos y de las mediaciones que rodean a objetos de relevancia social. Las representaciones que se estructuran en y desde las subjetividades en torno a los fenómenos de comunicación son, principalmente, una vía eficaz para acceder no sólo a las visiones respecto a los intercambios comunicativos, sino también a las habilitaciones y constricciones que se constituyen para la interacción con y a través de los medios.

\section{Referencias}

Abric, Jean (2001), "Prácticas sociales, representaciones sociales", en Abric, Jean [comp.], Prácticas Sociales y representaciones, México: Ediciones Coyoacán.

Banchs, María-Auxiliadora (1984), Concepto de representaciones sociales. Análisis comparativo, Venezuela: Universidad Central de Venezuela. 
Banchs, María-Auxiliadora (1990), "Las representaciones sociales: sugerencias sobre una alternativa teórica y un rol posible para los psicólogos sociales en Latinoamérica”, en Banchs, María-Auxiliadora [ed.], Aportes criticos a la psicología en Latinoamérica, Venezuela: Universidad Central de Venezuela.

Berger, Peter y Luckmann, Thomas (1986), La construcción social de la realidad, Argentina: Amorrortu.

Bertoldo, Raquel et al. (2016), Risks as a point of view: Scientists' social representations of nanotechnology, Reino Unido: Society for Risk Analysis, Europe, Jun, Bath.

Blumer, Herbert (1969), Symbolic Interactionism: Perspective and Method, Estados Unidos: Prentice-Hall.

Booth, Andrew et al. (2016), Systematic Approaches to a Successful Literature Review, Inglaterra: Sage.

Bourdieu, Pierre et al. (1968), El oficio del sociólogo: presupuestos epistemológicos, México: Siglo XXI.

Brunel, Maité et al. (2017), "Is the social representation of nanotechnology anchored in that of GMOs?”, en Journal of Risk Research. Disponible en: http://dx.doi.org/10.1080/13 669877.2017.1304976 [18 de abril de 2017].

Castorina, José-Antonio y Barreiro, Alicia (2012), "Los usos de las representaciones sociales en la investigación educativa”, en Educación, Lenguaje y Sociedad, vol. 9, núm. 9, Argentina: Universidad Nacional de la Pampa.

Da Silva (2011), G.H. Mead. A Reader, Reino Unido: Routledge.

De Castro, Emanuella et al. (2016), “Theory of Social Representations: Educational Possibilities in the Development of Active Methodologies for Significant Learning", en International Archives of Medicine, vol. 9, Estados Unidos: International Medical Society.

Doise, Willem (1985), “Les représentations sociales: définition d'un concept. / Social representations: Definition of the concept", en Connexions, vol. 45, Estados Unidos: New Mexico Tech and the University of Central Arkansas.

Doise, Willem (1991), "Las representaciones sociales: presentación de un campo de investigación”, en Anthropos, núm. 27, España: Anthropos.

Doise, Willem (1993), "Debating social representations", en Breakwell, G. y Canter, D. [eds.], Empirical Approaches to Social Representations, Inglaterra: Oxford Science Publications.

Farr, Robert y Moscovici, Serge [eds.] (1984), Social Representations, Inglaterra: Cambridge University.

Flament, Claude (2001), "Estructura, dinámica y transformación de las representaciones sociales", en Abric, Jean [comp.], Prácticas Sociales y representaciones, México: Ediciones Coyoacán.

Fuentes-Navarro, Raúl (2001), "Exploraciones teórico-metodológicas para la investigación sociocultural de los usos de la Internet", en Fuentes, R. y Vassallo de Lopes, M. [comps.], Comunicación: campo y objeto de estudio. Perspectivas reflexivas latinoamericanas, México: Universidad de Guadalajara.

González-Rey, Fernando (2002), Sujeto y subjetividad: una aproximación histórico-cultural, México: Thomson.

Hall, Stuart (1993), “Culture, community, nation”, en Cultural Studies, vol. 7, núm. 3, Inglaterra: Taylor and Francis.

Harré, Rom (2002), La Epistemología de las representaciones sociales, Cuba: Dirección de Información Científica y Técnica, Universidad de La Habana. 
Rainer Rubira-García y Belén Puebla-Martínez. Representaciones sociales y comunicación: apuntes teóricos para un diálogo interdisciplinar inconcluso

Ibáñez, Tomás (1988), Ideologias de la vida cotidiana. Psicología de las representaciones sociales, España: Sendai.

Islas, Claudia (2016), "Representaciones sociales de estudiantes universitarios acerca del blearning: un análisis de contenido”, en RED. Revista de Educación a Distancia, núm. 51, España: Universidad de Murcia. Disponible en: http://dx.doi.org/10.6018/red/51/6 [18 de abril de 2017].

Jodelet, Denise (1986), "La representación social: fenómenos, concepto y teoría”, en Moscovici, Serge [comp.], Psicología Social II. Pensamiento y vida social. Psicología social y problemas sociales, España: Paidós.

Machi, Lawrence y McEvoy, Brenda (2016), The literature review: six steps to success, Estados Unidos: Thousand Oaks, Corwin.

Martín-Barbero, Jesús (1987), De los medios a las mediaciones. Comunicación, cultura y hegemonía, México: Gili.

Martín-Barbero, Jesús (2001), Al sur de la modernidad. Comunicación, globalización y multiculturalidad, Estados Unidos: Nuevo Siglo.

Martín-Serrano, Manuel (2004), La producción social de comunicación, España: Alianza.

Moscovici, Serge (1985), "Introducción: El campo de la psicología social”, en Moscovici, Serge [comp.], Psicología Social I. Influencia y cambio de actitudes. Individuos y grupos, España: Paidós.

Moscovici, Serge y Hewstone, Miles (1986), “De la ciencia al sentido común”, en Moscovici, Serge [comp.], Psicología Social II. Pensamiento y vida social. Psicología social y problemas sociales, España: Paidós.

Moscovici, Serge y Hewstone, Miles (2013), Le scandale de la pensée sociale. Textes fondamentaux et inédits sur les représentations sociales réunis et introduits par $N$. Kalampalikis, Francia: Editions de l'EHESS.

Orozco, Guillermo (2002), "Mediaciones tecnológicasy des-ordenamientos comunicacionales”, en Signo y Pensamiento, núm. 41, vol. 21, Colombia: Facultad de Comunicación y Lenguaje, Universidad Javeriana.

Peirce, Charles (1986), La ciencia de la semiótica, Argentina: Nueva Visión.

Piçarra, N. et al. (2016), "Making sense of social robots: A structural analysis of the layperson's social representation of robots”, en Revue Européenne de Psychologie Appliquéel European Review of Applied Psychology, vol. 66, Francia: Elsevier.

Rodríguez, Tania (2009), "Sobre el potencial teórico de las representaciones sociales en el campo de la comunicación”, en Comunicación y sociedad, núm. 11, México: Universidad de Guadalajara. Disponible en: http://www.scielo.org.mx/scielo.php?script=sci_ arttext\&pid=S0188-252X2009000100002\&lng=es\&tlng=es [25 de noviembre de 2017].

Rosch, Eleanor (1977), "Classification of Real-World Objects: Origins and Representations in Cognition”, en Johnson-Laird, P. y Wason, P., Thinking: Readings in Cognitive Science, Inglaterra: Cambridge University Press.

Tomkins, Silvan (1962), Affect Imagery Consciousness. The Positive Effects, Inglaterra: Tavistock. Wagner, Wolfgang et al. (1999), "Theory and method of social representations", en Asian Journal of Social Psychology, núm.1, vol. 2, Inglaterra: Oxford, Blackwell Publishing, Asian Association of Social Psychology, Japanese Group Dynamics Association.

Wagner, Wolfgang y Hayes, N. (2005), Everyday Discourse and Common-Sense-The Theory of Social Representation, Estados Unidos: Palgrave Macmillan. 
Young, Rachel et al. (2016), "Social Representation of Cyberbullying and Adolescent Suicide: A Mixed-Method Analysis of News Stories”, en Communication, vol. 0, Inglaterra: Taylor and Francis.

Zubiri, Xavier (1994), Los problemas fundamentales de la metafísica occidental, España: Alianza.

Rainer Rubira-García. Doctor en Ciencias de la Comunicación por la Universidad Rey Juan Carlos, en Madrid, España. Licenciado en Comunicación Social por la Universidad de La Habana, Cuba; Máster en Comunicación y Problemas Socioculturales. Actualmente es investigador de la Cátedra UNESCO de Investigación en Comunicación de la Universidad Rey Juan Carlos y profesor visitante del propio centro. Líneas de investigación: redes sociales digitales, comunicación corporativa, estudios de Internet, género, comunicación política y comunicación internacional. Publicaciones recientes: Rubira, Rainer y Gil-Egui, Gisela, "Wikipedia as a space for discursive constructions of globalization”, en International Communication Gazette (2017); Núñez-Puente, S., Rubira-García, R. y Fernández-Romero, D., "Online Activism and Subject Construction of the Victim of Gender-based Violence on Spanish YouTube Channels: Multimodal Analysis and Performativity", en European Journal of Women's Studies, vol. 22, núm. 3, Inglaterra: Sage (2015); Santín, M. y Rubira, R., "Promotional Practices in News Programs: The Case of Spanish Public Television”, en International Journal of Communication, vol. 8, núm. 19, Estados Unidos: University of Southern California (2014).

Belén Puebla-Martínez. Doctora en Ciencias de la Comunicación por la Universidad Rey Juan Carlos. Profesora e investigadora de la URJC. Licenciada en Periodismo y en Comunicación Audiovisual por la misma universidad. Líneas de investigación: estudio de la ficción televisiva española, análisis de los métodos analíticos de investigación en comunicación social y estudio del uso de las redes sociales como forma de comunicación. Publicaciones recientes: Puebla Martínez, B. y Sierra Navarro, N., "Perspectivas de las teorías clásicas de la comunicación frente a las 'olvidadas' referencias a la actualidad en la telecomedia española”, en revista Index.comunicación, vol. 6, núm. 2, España: Universidad Rey Juan Carlos, ISSN: 2174-1859 (2016). Disponible en: http://journals.sfu.ca/indexcomunicacion/index. php/indexcomunicacion/article/view/224; González, L., Puebla, B. y Prieto, P., "El imaginario bélico a través de los carteles del cine norteamericano entre 1914 y 1918”, en L'Atalante revista de estudios cinematográficos, núm. 21, España: Associació Cinefórum L'Atalante, ISSN: 2340-6992 (2016). Disponible en: http://www.revistaatalante.com/index.php?journ 
al=atalante\&page=article\&op=view\&path\%5B\%5D=310; González, L., Puebla, B., Birkner, T. y Pérez, P., "El diseño periodístico como actor fundamental en los modelos de prensa popular. Una aproximación a las causas de cierre del diario Claro", en Revista Latina de Comunicación Social, núm. 70, España: Universidad de La Laguna, ISSN: 1138-5820 (2015). Disponible en: http://www.revistalatinacs.org/070/paper/1075/45es.html DOI: $10.4185 /$ RLCS-2015-1075.

Recepción: 21 de julio de 2017.

Aprobación: 28 de octubre de 2017. 
